\title{
Gambogic acid exerts cardioprotective effects in a rat model of acute myocardial infarction through inhibition of inflammation, iNOS and $\mathrm{NF}-\kappa \mathrm{B} / \mathrm{p38}$ pathway
}

\author{
DUAN NA, HOU AIJIE, LUAN BO, MIAO ZHILIN and YUAN LONG \\ Department of Cardiology, The People's Hospital of China Medical University, The People's \\ Hospital of Liaoning Province, Shenyang, Liaoning 110016, P.R. China
}

Received March 29, 2016; Accepted September 5, 2017

DOI: $10.3892 /$ etm.2017.5599

\begin{abstract}
Gamboge, the dried resin secreted by Garcinia maingayii (gambogic tree), was previously demonstrated to exert anti-inflammatory effects. The present study examined the effects of gambogic acid, the major active constituent of gamboge, on myocardial infarction (MI) and inflammation in a rat model and explored the possible underlying mechanisms. The results demonstrated that gambogic acid inhibited the ratio of heart weight to body weight and myocardial damage (via lactate dehydrogenase and cardiac troponin T) in rats with MI. Gambogic acid suppressed the activation of interleukin (IL)-6 and tumor necrosis factor- $\alpha$, and increased IL-10 levels in MI rats. Furthermore, gambogic acid reduced inducible nitric oxide synthase (iNOS), matrix metalloproteinase (MMP)-2, MMP-9, intercellular adhesion molecule-1 (ICAM-1), nuclear factor $(\mathrm{NF})-\kappa \mathrm{B} / \mathrm{p} 65$ and phosphorylated p38 protein in ischemic myocardial tissue of MI rats. In conclusion, gambogic acid exerted anti-inflammatory effects in MI rats by targeting the iNOS, MMPs, ICAM-1, NF- $\mathrm{B}$ and p38 pathways. Gambogic acid may protect against MI-induced inflammation in rats, which may be associated with the activation of the $\mathrm{NF}-\kappa \mathrm{B} / \mathrm{p} 38$ pathway.
\end{abstract}

\section{Introduction}

Acute myocardial infarction (AMI) is a heart condition that seriously threatens human health. With the change of people's lifestyle and an the aging of the population, cardiovascular diseases, particularly congestive heart failure and malignant arrhythmia caused by AMI, have gained a higher morbidity

Correspondence to: Dr Duan Na, Department of Cardiology, The People's Hospital of China Medical University, The People's Hospital of Liaoning Province, 33 Wenyi Road, Shenyang, Liaoning 110016 , P.R. China

E-mail: duanshuopu9956@126.com

Key words: gambogic acid, myocardial infarction, inflammation, $\mathrm{NF}-\kappa \mathrm{B}, \mathrm{p} 38$ and mortality on a global scale (1). Recently, with the extensive development of heart interventional operations and the rapid development of drug treatments for AMI treatment, myocardium that is close to becoming necrotic is effectively saved (2). The prognosis of AMI patients is greatly improved, but numerous patients cannot be subjected to revascularization for various reasons (3). Thus, myocardium undergoes irreversible necrosis, causing ventricular remodeling (VR), resulting in the deterioration of cardiac function and finally developing into cardiac failure (4). The occurrence of ventricular remodeling is closely linked to the prognosis of AMI. Therefore, effectively delaying and reversing AMI-induced ventricular remodeling is key in preventing cardiac failure $(2,4)$.

AMI is myocardial necrosis caused by acute and durative ischemia and anoxia. Ischemia and anoxia of cardiac muscle tissues and the increase of wall strain may induce an inflammatory response (5). In the inflammatory process of AMI, interleukin (IL)- 6 and tumor necrosis factor (TNF)- $\alpha$ have a major role. IL-6 regulates various biochemical processes, including the synthesis of liver C-reactive protein (CRP) and the acute response of the liver, and also stimulates myocardial cells to express intercellular adhesion molecule (ICAM-1), so as to increase adhesion of neutrophil granulocytes and release of oxygen radicals (6). Furthermore, TNF- $\alpha$ increases the chemotaxis of hemameba, damages endothelial cells directly and induces CRP synthesis to exert a certain role. The abovementioned inflammatory processes inevitably cause damage of myocardial cells or even myocardial fibrosis (7). The inflammatory response is has been reported to exert an important role in the occurrence and development of myocardial fibrosis (8). Products released by inflammatory cells, including arachidonic acid, may be catalyzed or catabolized by epoxidase and lipoxidase to generate bioactive substances such as epoxyarachidonic acid, leukotrienes and prostaglandin, several of which may also promote fibrosis (9).

At present, the specific pathogenesis of ventricular remodeling has remained to be fully elucidated. Nitric oxide (NO) has become a hotspot in cardiovascular research (10). In vivo, NO is mainly produced by NO synthase (NOS) acting on its substrate L-arginine. Inducible i) NOS expression was found to be increased during myocardial failure. Furthermore, iNOS expression was reported to be increased in rats with 
MI (11). Under these circumstances, NO synthesis is markedly increased, reducing cardiac function and increasing mortality.

Gamboge, the dried resin secreted by Garcinia maingayii (gambogic tree) has effects of breaking blood and removing stasis according to principles of Traditional Chinese Medicine, as well as detoxification, stopping bleeding and killing insects, and is used for treating multiple diseases, including cancer and encephaledema. Gamboge is composed of $70-80 \%$ resin and $15-25 \%$ gum and its major constituents include gambogic acid, neogambogic acid, allogambogic acid, morellin, isomorellin, morellic acid and isomorellic acid. Gambogic acid is the major active component (12). The anti-tumor effects of gambogic acid are extensive and include the induction of tumor cell apoptosis, inhibition of the cell cycle, and an influence on the expression of certain oncogenes, cancer suppressor genes and associated proteins (13). As an anti-tumor drug, gambogic acid has an obvious inhibitory effect on the growth of tumors grown in animal models, but anti-tumor drugs often generate untoward effects and easily cause irreversible damage to the hemopoietic and immune system (14). In the present study, a rat model of AMI was used to investigate the protective effects of gambogic acid and their possible association with inhibition of inflammation via the iNOS and nuclear factor $(\mathrm{NF})-\kappa \mathrm{B} / \mathrm{p} 38$ pathways.

\section{Materials and methods}

Animals and drug. Six week-old male Sprague Dawley rats were obtained from the Animal Experimental Center of Dalian Medical University (Dalian, China). Rats were housed under standard conditions $\left(22-24^{\circ} \mathrm{C}, 55-60 \%\right.$ humidity) and supplied with drinking water and food ad libitum. The animal protocol of this study was approved by the Institutional Animal Care and Use Committee of Guangzhou Medical University (Guangzhou, China). Gambogic acid (powder, $\geq 95 \%$ pure according to high-performance liquid chromatography; Fig. 1) was from Sigma-Aldrich (Merck KGaA, Darmstadt, Germany).

Induction of AMI and group design. All experimental rats were randomly divided into 3 groups as follows: Sham group $(n=6)$, vehicle-treated group $(n=8)$ and gambogic acid-treated group $(n=8)$. In the gambogic acid-treated group, $24 \mathrm{~h}$ after the induction of the AMI model, gambogic acid $(1 \mathrm{mg} / \mathrm{kg})$ was intraperitoneally injected into AMI rats for 5 days. After being intraperitoneally anesthetized using sodium pentobarbitone (40 mg/kg; Sigma-Aldrich; Merck KGaA, Darmstadt, Germany), the rats were operated to ligate the left anterior descending coronary artery. When regional cyanosis appeared on the myocardial surface in AMI rats, the rat model of AMI was confirmed to be successful established. The rats were then weighed and sacrificed using excess sodium pentobarbitone after treatment with gambogic acid for 5 days. The heart of every rat was weighed and the heart weight $(\mathrm{mg})$ to body weight $(\mathrm{g})$ ratio $(\mathrm{HW} / \mathrm{BW})$ was calculated.

Measurement of lactate dehydrogenase (LDH), cardiac troponin (cTnT), TNF- $\alpha, I L-6$ and IL-10 levels. Blood was extracted after treatment with gambogic acid for 5 days from the vena cava of every rat and then centrifuged at $2,000 \mathrm{xg}$

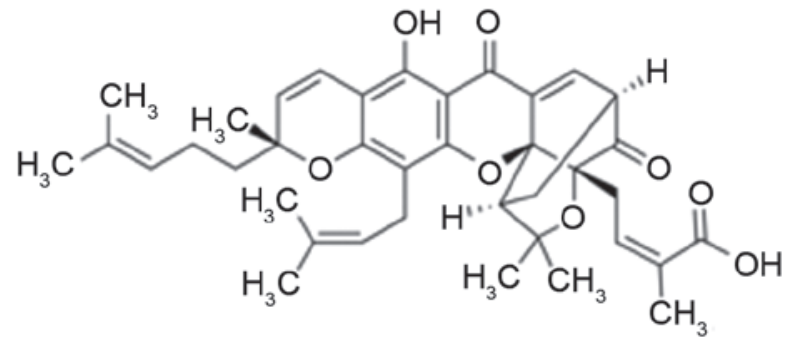

Figure 1. Chemical structure of gambogic acid.

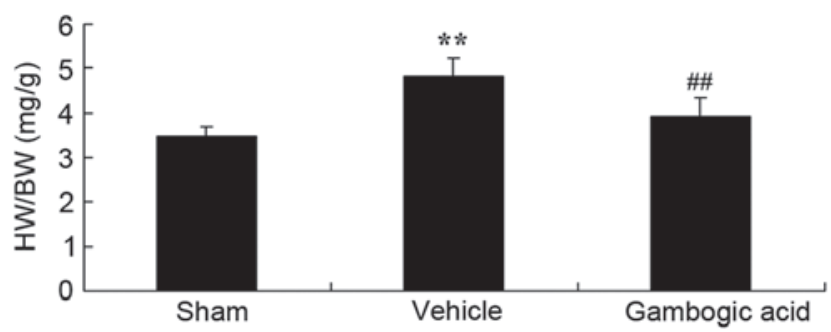

Figure 2. Effects of gambogic acid on the HW/BW in a rats model of myocardial infarction. ${ }^{* *} \mathrm{P}<0.01$ compared with control group; ${ }^{\# /} \mathrm{P}<0.01$ compared with vehicle group. $\mathrm{HW} / \mathrm{BW}$, heart weight to body weight ratio.

for 10 min to obtain serum. LDH (A020-2) and cTnT (H149-2) levels were determined using commercial kits (Nanjing Institute of Biological Engineering, Shanghai, China) according to the manufacturer's instructions. TNF- $\alpha$ (E-EL-R0019c), IL-6 (E-EL-R0896c) and IL-10 (E-EL-R0016c) levels were analyzed using ELISA kit (Elabscience Biotechnology Co., Ltd., Wuhan, China) according to the manufacturer's instructions.

Western blot analysis. Total protein was extracted from heart tissues of every rat and homogenized using radioimmunoprecipitation assay lysis buffer (Beyotime Institute of Biotechnology, Haimen, China) at $4^{\circ} \mathrm{C}$ for $30 \mathrm{~min}$ and the lysate was centrifuged at $2,000 \mathrm{x}$ g for $10 \mathrm{~min}$. The quantity of protein in the supernatant was measured using the bicinchoninic acid method (Beyotime Institute of Biotechnology). Protein (50 $\mu \mathrm{g}$ per lane) was resolved by $12 \%$ SDS-PAGE and then transferred onto polyvinylidene difluoride membranes (Bio-Rad Laboratories, Inc., Hercules, CA, USA). The membranes were probed with anti-iNOS (sc-649, 1:500), anti-MMP-2 (sc-10736, 1:500), anti-MMP-9 (sc-10737, 1:500), anti-ICAM-1 (sc-7891, 1:500), anti-NF-кB/p65 (sc-109, 1:500), anti-total p38 (sc-535, 1:500), anti-phosphorylated (p)-p38 (sc-101759, 1:500) and anti-GAPDH (sc-25778, 1:500; all from Santa Cruz Biotechnology, Inc., Dallas, TX, USA) antibodies overnight at $4^{\circ} \mathrm{C}$, followed by incubation with the goat anti-rabbit IgG-horseradish peroxidase secondary antibodies (sc-2004, 1:5,000; Santa Cruz Biotechnology, Inc.) at room temperature for $1 \mathrm{~h}$. Membranes was visualized with enhanced chemiluminescence (ECL)-Plus reagent ECL Western Blotting Substrate (Pierce; Thermo Fisher Scientific, Inc., Waltham, MA, USA).

Statistical analyses. Values are expressed as the mean \pm standard deviation and were analyzed using SPSS 17.0 software (SPSS, Inc., Chicago, IL, USA). Data were analyzed 

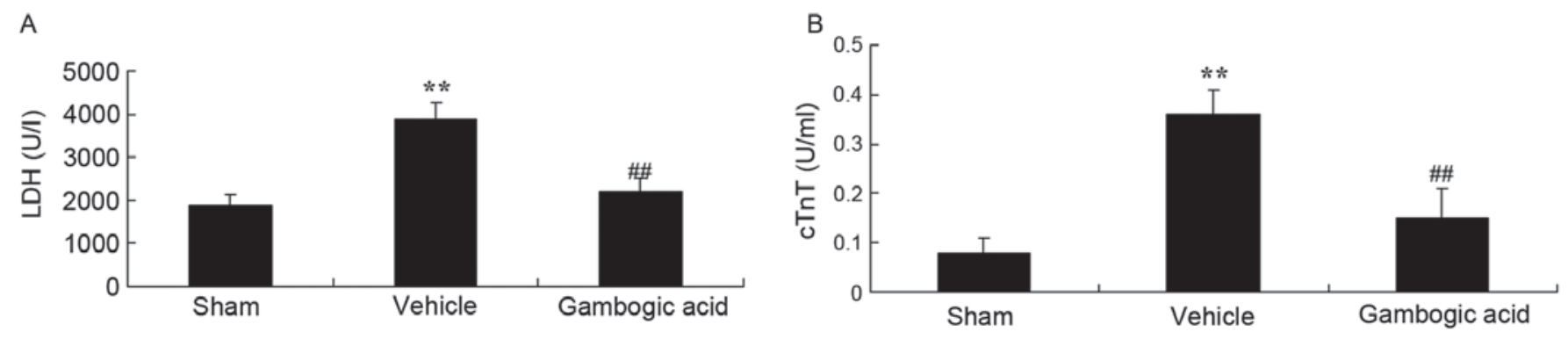

Figure 3. Effects of gambogic acid on myocardial damage in MI rats. (A) LDH and (B) cTnT levels in MI rats. ${ }^{* *} \mathrm{P}<0.01$ compared with control group; ${ }^{* \#} \mathrm{P}<0.01$ compared with vehicle group. MI, myocardial infarction; LDH, lactate dehydrogenase; cTnT, cardiac troponin.
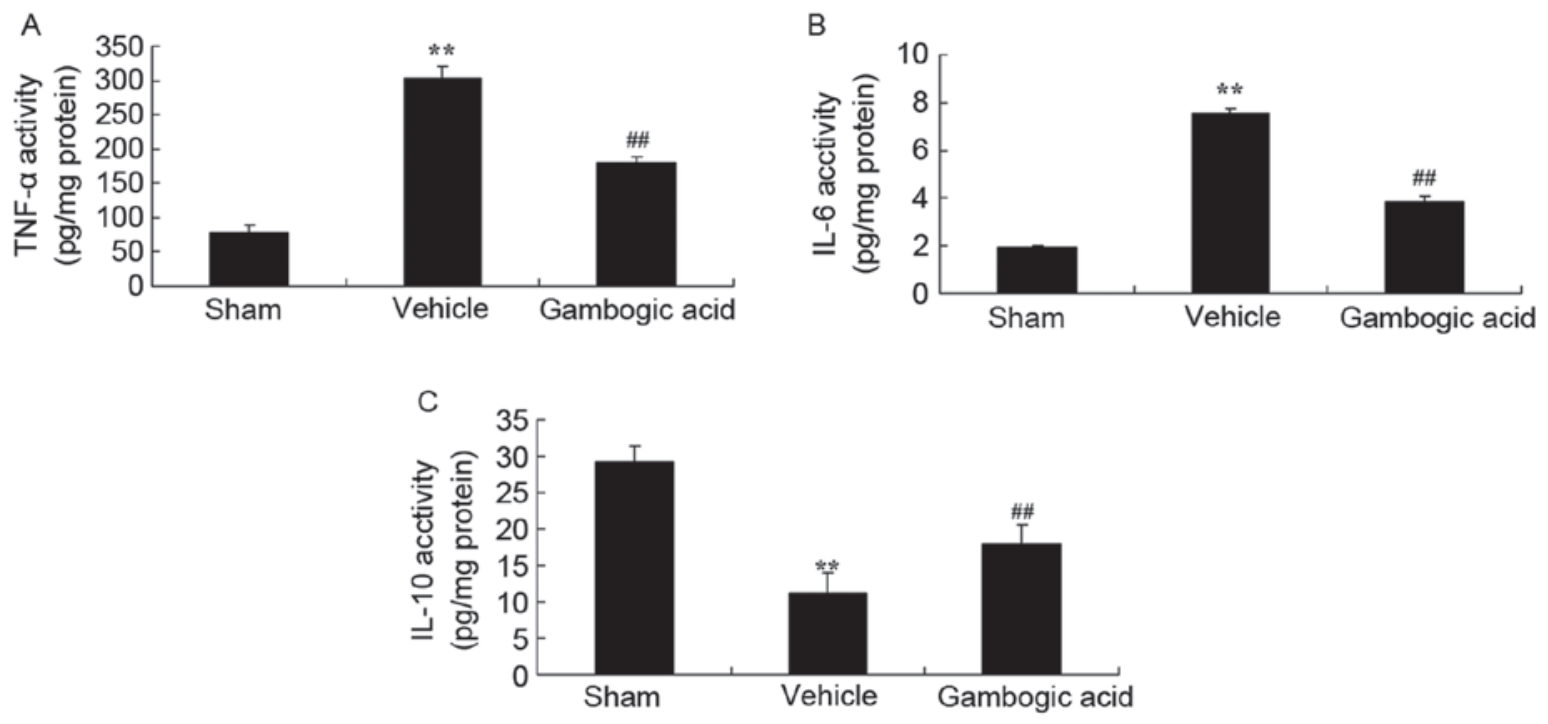

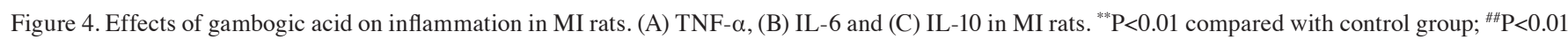
compared with vehicle group. MI, myocardial infarction; IL, interleukin; TNF, tumor necrosis factor.

by analysis of variance followed by the Student-Newman-Keuls test for multiple comparisons. $\mathrm{P}<0.05$ was considered to indicate a statistically significant difference.

\section{Results}

Gambogic acid inhibits AMI-induced increases in the $H W / B W$ ratio in rats. To investigate the effects of gambogic acid on the $\mathrm{HW} / \mathrm{BW}$ ratio under AMI conditions, AMI rats were injected with $1 \mathrm{mg} / \mathrm{kg}$ gambogic acid for 5 days at day 1 following surgery. As presented in Fig. 2, there was a significant increase in the HW/BW of AMI model rats compared with that in the control rats. Furthermore, gambogic acid significantly inhibited the AMI-associated increase in the HW/BW ratio of the rats (Fig. 2).

Gambogic acid reduces myocardial damage in MI rats. To investigate the inhibitory effects of gambogic acid on myocardial damage in MI rats, LDH and cTnT levels were measured with commercial kits. As presented in Fig. 3, these LDH and cTnT levels in the AMI model rats were markedly elevated compared with those in the control rat group. Of note, gambogic acid significantly inhibited the increase of LDH and cTnT levels in AMI rats as compared with that in the AMI model group (Fig. 2).
Gambogic acid reduces inflammation in MI rats. Inhibition of inflammation by gambogic acid was determined by assessing serum TNF- $\alpha$, IL- 6 and IL-10 with ELISA kits in the experimental groups. As displayed in Fig. 4A and B, the serum TNF- $\alpha$ and IL- 6 levels were obviously increased in the AMI model rats compared with those in the control group. Furthermore, a significant reduction of IL-10 levels in AMI model rats compared with those in the control group was observed (Fig. 4C). Of note, treatment with gambogic acid significantly inhibited AMI-associated increases in TNF- $\alpha$ and IL-6 levels as well as decreases in IL-10 levels (Fig. 4A-C).

Gambogic acid reduces iNOS protein expression in cardiac tissues of MI rats. To investigate the underlying mechanism of the protective effects of gambogic acid against AMI, iNOS protein expression in cardiac tissues of MI rats was assessed using western blot analysis. Compared with that in the control group, iNOS protein expression was significantly induced in AMI rats (Fig. 5). However, treatment with gambogic acid significantly suppressed the activation of the protein expression of iNOS in AMI rats as compared with that in the AMI model group (Fig. 5).

Gambogic acid reduces MMP-2 and MMP-9 protein expression in cardiac tissues of MI rats. To further 
A

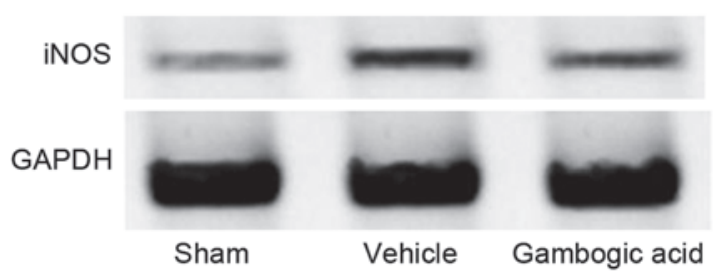

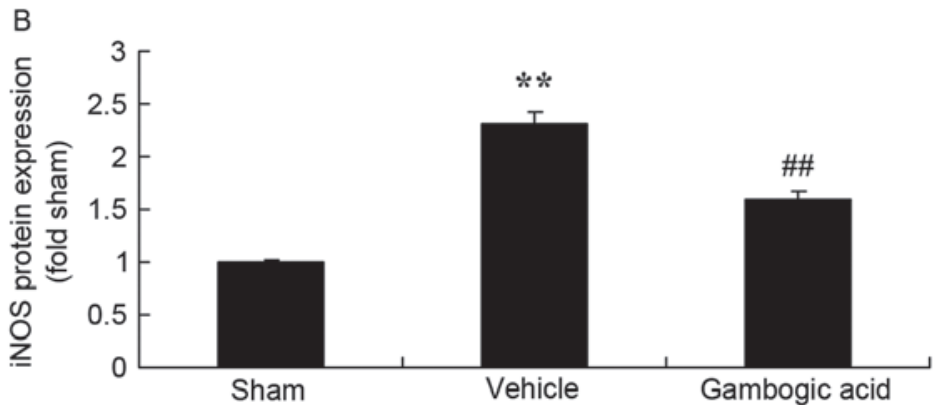

Figure 5. Effects of gambogic acid on iNOS protein expression in rats with myocardial infarction. (A) Representative western blot images of iNOS protein and (B) quantified expression levels. ${ }^{* *} \mathrm{P}<0.01$ compared with control group; ${ }^{\# \prime} \mathrm{P}<0.01$ compared with vehicle group. iNOS, inducible nitric oxide synthase.
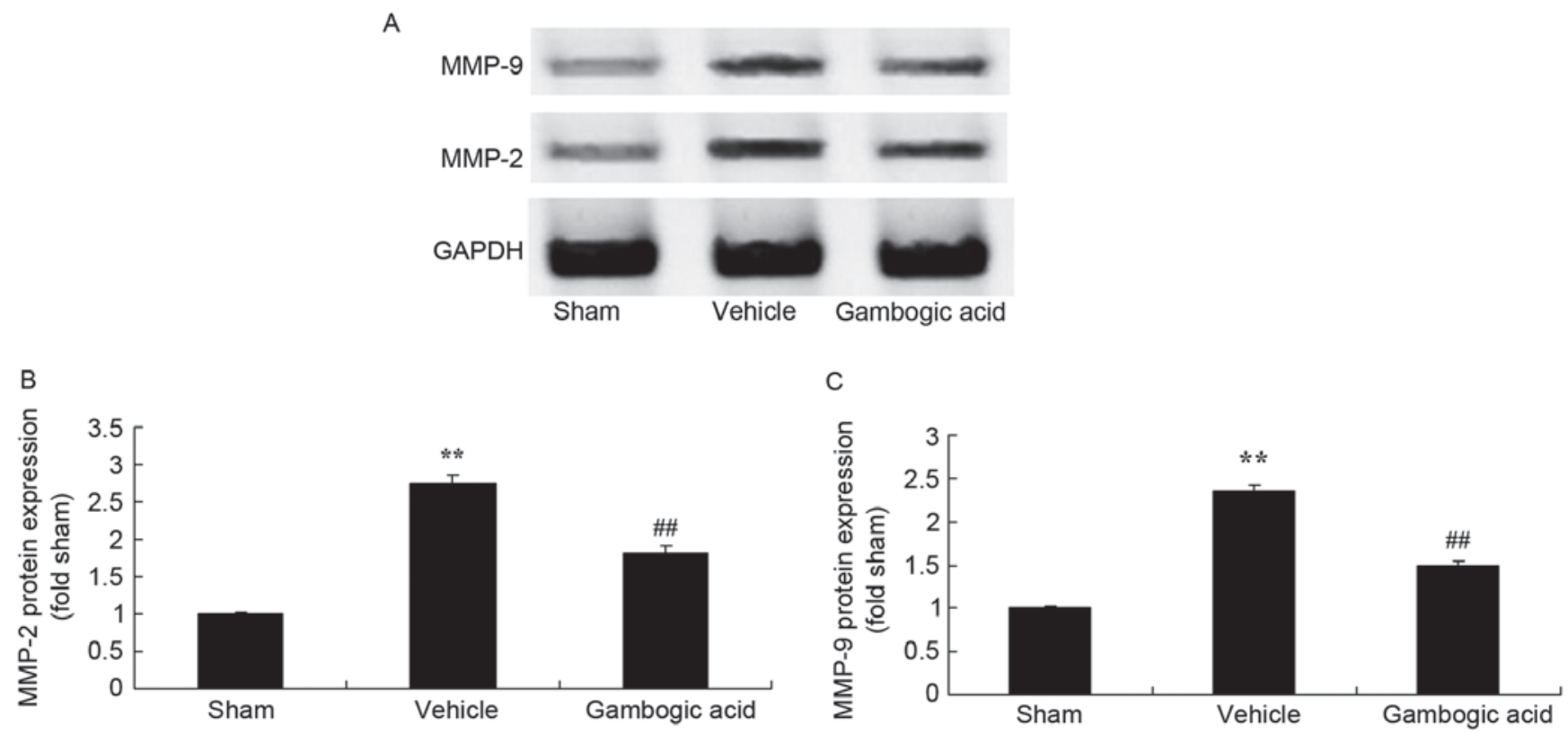

Figure 6. Effects of gambogic acid on MMP-2 and MMP-9 protein expression in rats with myocardial infarction. (A) Representative western blot images of MMP-2 and MMP-9 protein and (B and C) quantified expression levels. ${ }^{* *} \mathrm{P}<0.01$ compared with control group; ${ }^{\# \#} \mathrm{P}<0.01$ compared with vehicle group. MMP, matrix metalloproteinase.

investigate the involvement of MMP-2 and MMP-9 in the protective effect of gambogic acid on MI, MMP-2 and MMP-9 protein expression cardiac tissues of rats from each group was detected using western blot analysis. As presented in Fig. 6, MMP-2 and MMP-9 protein expression was significantly increased in AMI model rats compared with that in the control group. Of note, treatment with gambogic acid significantly inhibited MMP-2 and MMP-9 protein expression in AMI rats as compared with that in the AMI model group (Fig. 6).

Gambogic acid reduces ICAM-1 protein expression in cardiac tissues of MI rats. Furthermore, the present study investigated ICAM-1 protein expression in cardiac tissues of MI rats treated with gambogic acid using western blot analysis. As displayed in Fig. 7, a significant induction of ICAM-1 protein expression in AMI rats compared with the control group was observed. However, gambogic acid treatment significantly suppressed the protein expression of ICAM-1 in AMI rats compared with that in the AMI model group (Fig. 7).
Gambogic acid reduces $N F-\kappa B / p 65$ protein expression in cardiac tissues of MI rats. The present study further assessed the mechanism of the anti-inflammation effect of gambogic acid in MI rats by determining the protein expression of $\mathrm{NF}-\mathrm{\kappa B} / \mathrm{p} 65$ in their cardiac tissues using western blot analysis. As presented in Fig. 8, AMI significantly activated NF- $\mathrm{kB} / \mathrm{p} 65$ protein expression in AMI model rats compared with the control group. Gambogic acid treatment

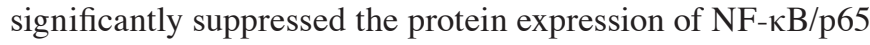
in AMI rats compared with that in the AMI model group (Fig. 8).

Gambogic acid reduces p38 activation in cardiac tissues of MI rats. To assess the involvement of $\mathrm{p} 38$ in MI rats, western blot analysis was used to assess the levels of p38 and its activated form, p-p38 in cardiac tissues. As illustrated in Fig. 9, the p-p38/p38 ratio in AMI model rats was significantly increased compared with that in the control group. However, gambogic acid significantly suppressed the levels of p-p38, leading to a reduced p-p38/p38 ratio in AMI model rats compared with that in the AMI model group. 
A

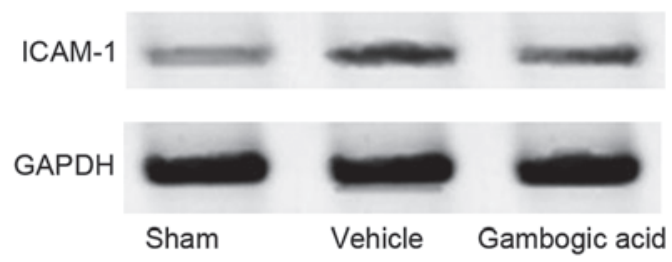

B

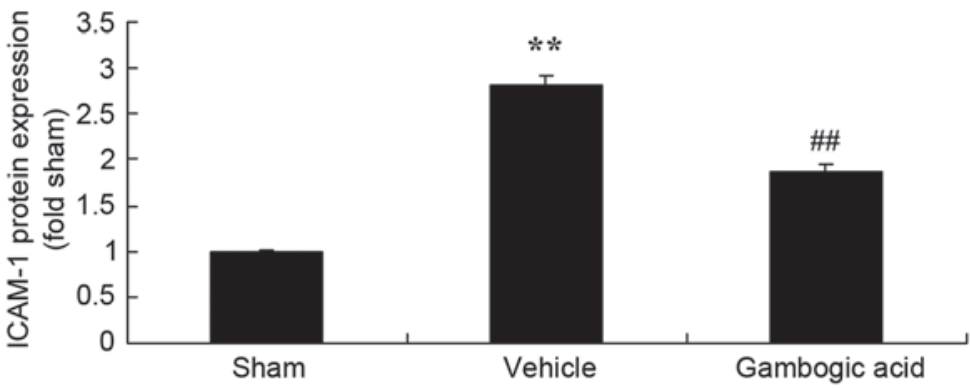

Figure 7. Effects of gambogic acid on ICAM-1 protein expression in rats with myocardial infarction. (A) Representative western blot images of ICAM-1 protein and (B) quantified expression levels. ${ }^{* *} \mathrm{P}<0.01$ compared with control group; ${ }^{\# \#} \mathrm{P}<0.01$ compared with vehicle group. ICAM-1, intercellular adhesion molecule-1.

A

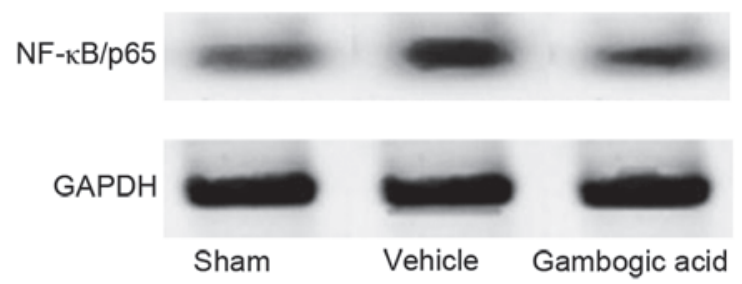

B

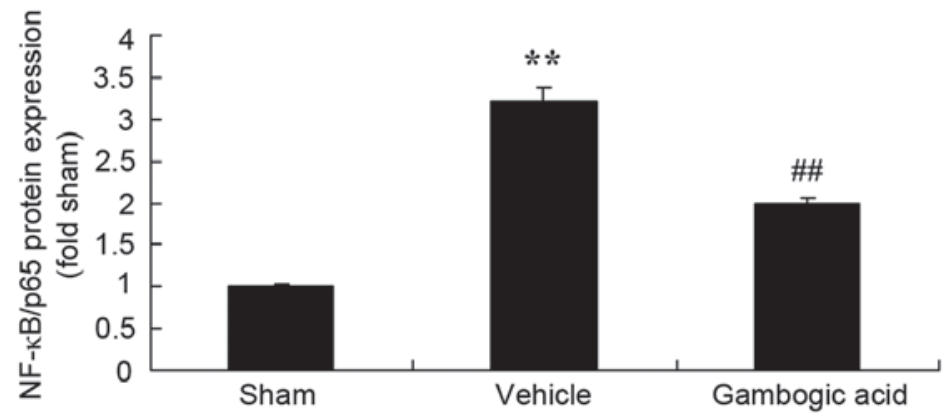

Figure 8. Effects of gambogic acid on NF- $\mathrm{kB} / \mathrm{p} 65$ protein expression in rats with myocardial infarction. (A) Representative western blot images of NF- $\mathrm{kB} / \mathrm{p} 65$ protein expression and (B) quantified expression levels. ${ }^{* * *} \mathrm{P}<0.01$ compared with control group; ${ }^{\# \#} \mathrm{P}<0.01$ compared with vehicle group. $\mathrm{NF}$, nuclear factor.

A

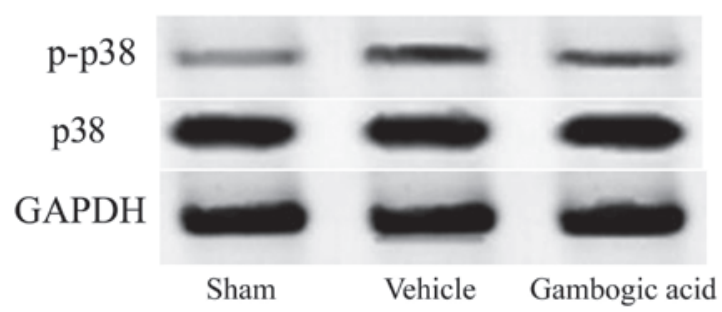

B

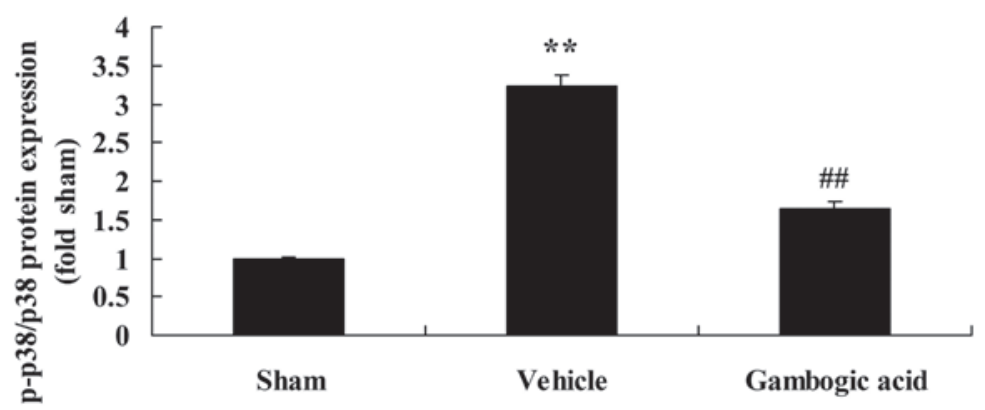

Figure 9. Effects of gambogic acid on p-p38 protein expression in rats with myocardial infarction. (A) Representative western blot images of p-p38 and p38 protein expression and (B) the quantified p-p38/p38 ratio. ${ }^{* *} \mathrm{P}<0.01$ compared with control group; ${ }^{\# \#} \mathrm{P}<0.01$ compared with vehicle group. $\mathrm{p}$-p38, phosphorylated p38.

\section{Discussion}

AMI is a serious coronary heart condition and AMI-associated ventricular remodeling is an important factor reducing the quality of life and survival rate of affected patients (15). The broad concept of AMI-associated ventricular remodeling implies that AMI is impacted by haemodynamics and non-haemodynamics, resulting in changes in heart size, shape and ultrastructure as well as damage of heart function (16). Ventricular remodeling is an important pathophysiological process of heart failure developed through AMI, occurs throughout the entire course of the disease and is one of the major factors impacting the immediate and long-term prognosis of patients after AMI (17). While ventricular remodeling occurs via multiple mechanisms, it is widely recognized that inflammation is an important cause of ventricular remodeling after MI and constitutes the basic mechanism of chronic cardiac failure development (5). In the present study, gambogic acid significantly inhibited the activation of HW/BW as well as increases in LDH and cTnT levels in cardiac tissues of AMI model rats.

After abrupt occlusion, the resulting MI and necrosis inevitably cause multiple inflammatory cells and mononuclear leucocytes to move to the infarct area. During the process, certain signaling pathways are activated in myocardial cells, so as to induce secretory cells of the heart to release multiple proteases and cause local inflammation-induced responses (8). Furthermore, during avascular necrosis, cardiac muscle tissues release or expose multiple autoantigens. Mechanical distraction and nervous activation finally result in increased secretion of 
cell factors, such as IL-6, IL-10 and TNF- $\alpha$ (18). These inflammatory factors not only have important roles in the myocardial damage process, but also participate in myocardial fibrosis and the ventricular remodeling process after the infarct (19). In the present study, it was observed that gambogic acid significantly inhibited TNF- $\alpha$ and IL-6 levels and enhanced IL-10 levels in the serum of AMI rats. Geng et al (12) indicated that gambogic acid protects from endotoxin shock and suppressed pro-inflammatory factors, which was in accordance to the findings of the present study.

In the scar formation process subsequent to an infarct, even if the front and back loads are slightly increased, adverse effects on future ventricular size and formation occur (20). Therefore, reduction of cardiac function caused by NO overproduction induced by iNOS and an obvious increase of the left ventricular end-diastolic pressure in ventricular remodeling after the infarct have an important effect on its occurrence and development. In the left ventricular remodeling process of AMI, regulation of a series of neurohumor factors have an important role (11). The renin-angiotensin system has the biggest influence on remodeling. AMI angiotensin-11 and $\alpha$-receptor activity increases may promote iNOS expression in myocardial cells, while increases of iNOS reduce cardiac function and output, and activate the renin-angiotensin system (10). If heart failure occurs, the activation of these factors may have persisted for a long time and participated in stimulating cardiac fibrosis, proliferation of blood vessels, as well as hypertrophy and compensatory expansion of the myocardium (11). The present study found that gambogic acid significantly suppressed the activation of the protein expression of iNOS in cardiac tissues of AMI rats.

During ventricular remodeling, activation of neurohumoral control and inflammation as well as changes of myocardial cells and intercellular substance structure caused by cell factors occur. AMI leads to remodeling of cardiac structure and functional alterations in line with a certain pattern, and expansion as well as changes in the shape of the ventriculus sinister occur, including changes of ventricular volume, shape, wall thickness and myocardial structure, resulting in an abnormal cardiac shape and structure as well as haemodynamics; the left ventricle is progressively expanded and contraction is gradually reduced, finally resulting in heart failure and death (21). The degree of severity of AMI not only depends on the infarct size, but is also closely associated with MMPs secreted by infiltrated inflammatory cells, while remodeling of the infarcted myocardium is also closely linked to infarct size, inflammatory response after the infarct, and repair of infarcted and marginal myocardium (22). The present study found that treatment with gambogic acid significantly inhibited MMP-2 and MMP-9 protein expression in cardiac tissues of AMI rats. Park et al (14) suggested that gambogic acid decreased MMP-2, MMP-9 and $\mathrm{NF}-\kappa \mathrm{B}$ expression in SK-HEP1 cells.

Immune cells have an important role in the inflammatory response and repair after MI. The activated Toll-like receptor/IL-1 and complement cascade system promote the transformation of endothelial cells and fibroblast into pro-inflammatory phenotypes through the NF- $\kappa \mathrm{B}$ signaling pathway $(18,23)$. Activated endothelial cells secrete large amounts of chemotactic factors to increase cell adherence, making neutrophile granuloxytes and mononuclear leucocytes gather and infiltrate into the infarcted area (23). Neutrophile granuloxytes move along the endothelial cell surface, activating integrin and adhesion molecules, and combining with endothelial cells through ICAM-1. After neutrophile granulocytes, mononuclear leucocytes and lymphocytes start to infiltrate into the infarcted tissue (24). In the present study, gambogic acid treatment significantly suppressed the protein expression of ICAM-1 and NF- $\mathrm{BB} / \mathrm{p} 65$ in cardiac tissues of AMI rats. This was in line with a study by Wen et al (25) suggesting that gambogic acid exhibits anti-psoriatic efficacy via ICAM-1.

Activation of p38 mitogen-activated protein kinase (MAPK) in AMI may result in increased expression of inflammatory factors (26). Numerous studies have demonstrated that restraining p38 MAPK during long-term myocardial ischemia obviously reduces the occurrence of MI and death (26-28). The mechanism involves the reduction of ischemic damage of the myocardium by restraining the generation of inflammatory factors including TNF- $\alpha$, IL-1 and IL-8 (29). The present study demonstrated that gambogic acid significantly suppressed p-p38 protein levels and the p-p38/p38 ratio in the cardiac tissues of AMI model rats. In line with this, Lu et al (30) suggested that gambogic acid inhibits angiogenesis via extracellular signal-regulated kinase, AKT and p38.

In conclusion, the present study suggested that gambogic acid significantly inhibited increases of the $\mathrm{HW} / \mathrm{BW}$ ratio as well as LDH and cTnT levels in the cardiac tissues of AMI model rats. The anti-inflammation effect of gambogic acid was possibly mediated via a block of the NF- $\kappa \mathrm{B} / \mathrm{p} 65$ signaling pathway, resulting in the inhibition of MMP-2, MMP-9, ICAM-1 and p38 MAPK signaling pathways. The results confirmed the cardioprotective effect of gambogic acid and suggested its potential application in the management of AMI-associated diseases.

\section{References}

1. Seo GW, Kim DK, Kim KH, Seol SH, Jin HY, Yang TH, Ahn Y, Jeong MH, Song PS and Kim DI; Other Korea Acute Myocardial Infarction Registry Investigators: Impact of carvedilol versus $\beta 1$-selective $\beta$ blockers (bisoprolol, metoprolol, and nebivolol) in patients with acute myocardial infarction undergoing percutaneous coronary intervention. Am J Cardiol 116: 1502-1508, 2015.

2. Holme I, Szarek M, Cater NB, Faergeman O, Kastelein JJ, Olsson AG, Tikkanen MJ, Larsen ML, Lindahl C and Pedersen TR; Incremental Decrease in End Points Through Aggressive Lipid Lowering Study Group: Adherence-adjusted efficacy with intensive versus standard statin therapy in patients with acute myocardial infarction in the IDEAL study. Eur J Cardiovasc Prev Rehabil 16: 315-320, 2009.

3. Parodi G, Valenti R, Migliorini A, Maehara A, Vergara R, Carrabba N, Mintz GS and Antoniucci D: Comparison of manual thrombus aspiration with rheolytic thrombectomy in acute myocardial infarction. Circ Cardiovasc Interv 6: 224-230, 2013.

4. Wallander M, Norhammar A, Malmberg K, Ohrvik J, Rydén L and Brismar K: IGF binding protein 1 predicts cardiovascular morbidity and mortality in patients with acute myocardial infarction and type 2 diabetes. Diabetes Care 30: 2343-2348, 2007.

5. Doi M, Nosaka K, Miyoshi T, Iwamoto M, Kajiya M, Okawa K, Nakayama R, Takagi W, Takeda K, Hirohata S and Ito H: Early eicosapentaenoic acid treatment after percutaneous coronary intervention reduces acute inflammatory responses and ventricular arrhythmias in patients with acute myocardial infarction: A randomized, controlled study. Int J Cardiol 176: 577-582, 2014.

6. TRIUMPH Investigators, Alexander JH, Reynolds HR, Stebbins AL, Dzavik V, Harrington RA, Van de Werf F and Hochman JS: Effect of tilarginine acetate in patients with acute myocardial infarction and cardiogenic shock: The TRIUMPH randomized controlled trial. JAMA 297: 1657-1666, 2007. 
7. Liu HL, Yang Y, Yang SL, Luo JP, Li H, Jing LM and Shen ZQ: Administration of a loading dose of atorvastatin before percutaneous coronary intervention prevents inflammation and reduces myocardial injury in STEMI patients: A randomized clinical study. Clin Ther 35: 261-272, 2013.

8. Sun SJ, Wu XP, Song HL and Li GQ: Baicalin ameliorates isoproterenol-induced acute myocardial infarction through iNOS, inflammation, oxidative stress and P38MAPK pathway in rat. Int J Clin Exp Med 8: 22063-22072, 2015.

9. Melloni C, Sprecher DL, Sarov-Blat L, Patel MR, Heitner JF, Hamm CW, Aylward P, Tanguay JF, DeWinter RJ, Marber MS, et al: The study of LoSmapimod treatment on inflammation and InfarCtSizE (SOLSTICE): Design and rationale. Am Heart J 164: 646-653.e3, 2012.

10. Marfella R,Di Filippo C, Portoghese M,Ferraraccio F, Rizzo MR, Siniscalchi M, Musacchio E, D'Amico M, Rossi F and Paolisso G: Tight glycemic control reduces heart inflammation and remodeling during acute myocardial infarction in hyperglycemic patients. J Am Coll Cardiol 53: 1425-1436, 2009.

11. Chen H, Xu Y, Wang J, Zhao W and Ruan H: Baicalin ameliorates isoproterenol-induced acute myocardial infarction through iNOS, inflammation and oxidative stress in rat. Int J Clin Exp Pathol 8: 10139-10147, 2015.

12. Geng J, Xiao S, Zheng Z, Song S and Zhang L: Gambogic acid protects from endotoxin shock by suppressing pro-inflammatory factors in vivo and in vitro. Inflamm Res 62: 165-172, 2013.

13. Rahman MA, Kim NH and Huh SO: Cytotoxic effect of gambogic acid on SH-SY5Y neuroblastoma cells is mediated by intrinsic caspase-dependent signaling pathway. Mol Cell Biochem 377: 187-196, 2013

14. Park MS, Kim NH, Kang CW, Oh CW and Kim GD Antimetastatic effects of gambogic acid are mediated via the actin cytoskeleton and NF- $\mathrm{KB}$ pathways in SK-HEP1 cells. Drug Dev Res 76: 132-142, 2015.

15. Hofma SH, Smits PC, Brouwer J, Velders MA, van 't Hof AW, Queré M, de Vries CJ and van Boven AJ: Long-term follow-up of second-generation everolimus-eluting stents versus first-generation sirolimus-eluting stents in acute myocardial infarction: Three-year results of the XAMI trial. EuroIntervention 10 : $1280-1283,2015$

16. Beitnes JO, Hopp E, Lunde K, Solheim S, Arnesen H, Brinchmann JE, Forfang K and Aakhus S: Long-term results after intracoronary injection of autologous mononuclear bone marrow cells in acute myocardial infarction: The ASTAMI randomised, controlled study. Heart 95: 1983-1989, 2009.

17. Kim JS, Park SM, Kim BK, Ko YG, Choi D, Hong MK, Seong IW, Kim BO, Gwon HC, Hong BK, et al: Efficacy of clotinab in acute myocardial infarction trial-ST elevation myocardial infarction (ECLAT-STEMI). Circ J 76: 405-413, 2012.

18. De Jesus NM, Wang L, Herren AW, Wang J, Shenasa F, Bers DM, Lindsey ML and Ripplinger CM: Atherosclerosis exacerbates arrhythmia following myocardial infarction: Role of myocardial inflammation. Heart Rhythm 12: 169-178, 2015.

19. Sheu JJ, Lee FY, Yuen CM, Chen YL, Huang TH, Chua S, Chen YL, Chen CH, Chai HT, Sung PH, et al: Combined therapy with shock wave and autologous bone marrow-derived mesenchymal stem cells alleviates left ventricular dysfunction and remodeling through inhibiting inflammatory stimuli, oxidative stress \& enhancing angiogenesis in a swine myocardial infarction model. Int J Cardiol 193: 69-83, 2015.
20. Palaniyappan A, Uwiera RR, Idikio H, Menon V, Jugdutt C and Jugdutt BI: Attenuation of increased secretory leukocyte protease inhibitor, matricellular proteins and angiotensin II and left ventricular remodeling by candesartan and omapatrilat during healing after reperfused myocardial infarction. Mol Cell Biochem 376: 175-188, 2013.

21. Tan J and Hua Q: Correlations between serum inflammation factors and left ventricular remodeling in acute ST segment elevation myocardial infarction. Yonsei Med J 53: 501-507, 2012.

22. Li X, de Boer OJ, Ploegmaker H, Teeling P, Daemen MJ, de Winter RJ and van der Wal AC: Granulocytes in coronary thrombus evolution after myocardial infarction-time-dependent changes in expression of matrix metalloproteinases. Cardiovasc Pathol 25: 40-46, 2016.

23. Ueland T, Espevik T, Kjekshus J, Gullestad L, Omland T, Squire IB, Frøland SS, Mollnes TE, Dickstein K and Aukrust P: Mannose binding lectin and soluble Toll-like receptor 2 in heart failure following acute myocardial infarction. J Card Fail 12: 659-663, 2006

24. Andersen G $\varnothing$, Ueland T, Knudsen EC, Scholz H, Yndestad A, Sahraoui A, Smith C, Lekva T, Otterdal K, Halvorsen B, et al: Activin A levels are associated with abnormal glucose regulation in patients with myocardial infarction: Potential counteracting effects of activin A on inflammation. Diabetes 60: 1544-1551, 2011.

25. Wen J, Pei H, Wang X, Xie C, Li S, Huang L, Qiu N, Wang W, Cheng $X$ and Chen L: Gambogic acid exhibits anti-psoriatic efficacy through inhibition of angiogenesis and inflammation. J Dermatol Sci 74: 242-250, 2014.

26. Arabacilar P and Marber M: The case for inhibiting p38 mitogen-activated protein kinase in heart failure. Front Pharmacol 6: 102, 2015.

27. Wang M, Tsai BM, Reiger KM, Brown JW and Meldrum DR: 17-beta-Estradiol decreases p38 MAPK-mediated myocardial inflammation and dysfunction following acute ischemia. J Mol Cell Cardiol 40: 205-212, 2006.

28. Grossi V, Lucarelli G, Forte G, Peserico A, Matrone A, Germani A, Rutigliano M, Stella A, Bagnulo R, Loconte D, et al: Loss of STK11 expression is an early event in prostate carcinogenesis and predicts therapeutic response to targeted therapy against MAPK/p38. Autophagy 11: 2102-2113, 2015.

29. Li Z, Ma JY, Kerr I, Chakravarty S, Dugar S, Schreiner G and Protter AA: Selective inhibition of p38alpha MAPK improves cardiac function and reduces myocardial apoptosis in rat model of myocardial injury. Am J Physiol Heart Circ Physiol 291: H1972-H1977, 2006.

30. Lu N, Yang Y, You QD, Ling Y, Gao Y, Gu HY, Zhao L, Wang XT and Guo QL: Gambogic acid inhibits angiogenesis through suppressing vascular endothelial growth factor-induced tyrosine phosphorylation of KDR/Flk-1. Cancer Lett 258: 80-89, 2007. 